\title{
Feasibility Study on iPhone Accelerometer for Gait Detection
}

\author{
Herman K.Y. Chan, Huiru Zheng, Haiying Wang, Rachel Gawley, Mingjing Yang, Roy Sterritt \\ School of Computing and Mathematics \\ University of Ulster \\ Northern Ireland, UK \\ chan-h@email.ulster.ac.uk
}

\begin{abstract}
Falls amongst the elderly is becoming a major problem with over $50 \%$ of elderly hospitalizations due to injury from fall related accidents. Healthcare expenses are dramatically rising due to growing elderly population. Many current technologies for gait analysis are laboratory-based and can incur substantial costs for the healthcare sector for treatment of falls. However utilization of alternative commercially available technologies can potentially reduce costs. Accelerometers are one such option, being ambulatory motion sensors for the detection of orientation and movement. Smart mobile devices are considered as noninvasive and increasingly contain accelerometers for detecting device orientation. This study looks at the capabilities of the accelerometer within a smart mobile device, namely the iPhone, for identification of gait events from walking along a flat surface. The results prove that it is possible to extract features from the accelerometer of an iPhone such as step detection, stride time and cadence.
\end{abstract}

\section{INTRODUCTION}

In the UK, life expectancy in 2007-2008 has risen to 77.7 years and 81.9 years for male and female respectively compared to 73 years and 79 years in 1990 [1]. The percentage of injuries due to falls increase with ageing; $86 \%$ of injuries in the over $85 \mathrm{~s}$ are due to falls [2]. Up to one in three over $65 \mathrm{~s}$ suffers from a fall each year, costing up to $£ 4.6$ million a day, adding up to an estimated $£ 1.7$ billion per year [3].

These statistics and costs have resulted in a growing interest in the field of fall prevention. Gait analysis can assist by assessing a patient's walking pattern to underline abnormalities and potentially determine causes of injuries by abnormal gait. Gait analysis in the clinical environment requires specialists to assess patients with optical systems such as Codamotion [5], Vicon [6] and Qualisys [7]. Using these systems require spatial gait labs, manual settings and assessments by specialists.

Accelerometer and other inertial sensor technologies have been developed and applied to explore gait pattern changes and recognizing activities of a user. Accelerometers are relatively small and low-cost devices that provide quantitative measurements. There are many types of accelerometer that can measure single to multi axis to determine acceleration from different direction to sense orientation. In using the tri-axial output of the accelerometers, basic daily movements can be classified by measuring postural orientations. For example, $\operatorname{activPAL}^{\mathrm{TM}}$ [4] is an accelerometer based system for measuring the gross activity; however it does not provide in depth information such as features of gait. The IDEEA device [8] can provide a portable solution to gross activity and gait analysis. It can identify and differentiate between more than 40 types of activities providing 17 different parameters of gait such as single or double limb support, cycle, swing and step duration, ground impact, speed, cadence, step and stride length via LifeGait system. However, this system, similar to the activPAL ${ }^{\mathrm{TM}}$ device, still requires clinical professional's assist and it is expensive for individual users. Yang et al. has identified a subset of gait features extract from a standalone accelerometer for gait analysis [9]. Compared with standalone accelerometers, smart mobile phone is low cost and provides a user interface for ease of use.

Smart mobile phones have become widely available commercially over the past decade since their first appearance in the early 90s. Smart mobile devices provide a rich technology platform for users and developers to explore mobile computing possibilities. In recent years, smartphone manufacturers have adapted micro technologies such as micro electromechanical systems (MEMS) accelerometers and gyroscopes to determine device orientation. Potentially, the MEMS accelerometers in these devices can provide meaningful data of user's movement and may be useful for gait analysis, assessment and monitoring in replacing the conventional piezoelectric crystal based accelerometers which are considered to be large and clumsy. The objective of this research is to investigate the feasibility of using a smart mobile device to gather the acceleration raw data during free walking and analyses the data using the absolute distance algorithm. A smartphone based gait analysis system will be developed for supporting gait monitoring and assessment.

The remainder of the paper is organized as follows. In Section 2, we introduce methodology used in this research. Section 3 describes the results and the paper is concluded by discussion and summary in Section 4.

\section{METHODOLOGY}

The approach used in this study follows the workflow shown in Figure 1, which includes devices, design and development of device application, data collection and analysis of data gathered. 


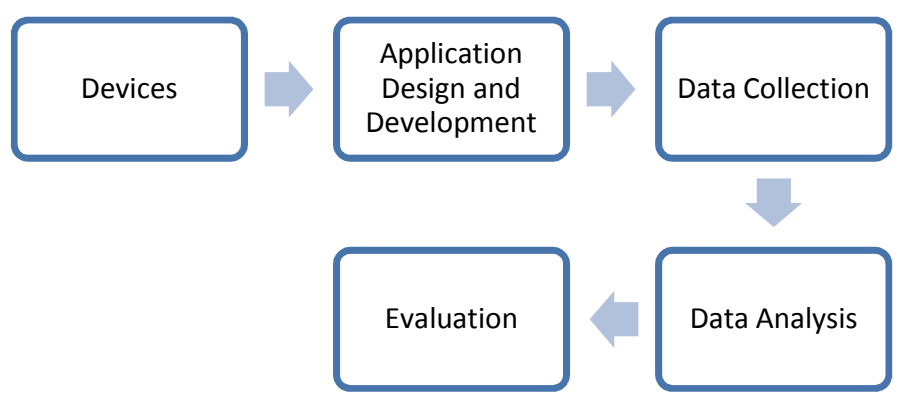

Fig 1. Workflow diagram of the research approach.

\section{A. Devices}

The device used in the study is an iPhone 3GS which contains an ultra-compact low-power axes linear accelerometer, the LIS302DL [10] MEMS accelerometer. It is capable of measuring acceleration data with data sampling rate of $100 \mathrm{~Hz}$. An accelerometer measures acceleration in g-force; $1 \mathrm{~g}$ is equal to 9.8 meters per second squared. The main functionality of an accelerometer in a mobile device is for screen orientation switching. The accelerometer within the device is capable of providing data on three different axes. The position to place to device is based on a common position in which a user would store a mobile device

An additional device that records a different type of data was also selected as a means of validating the results of the iPhone acceleration data. The wearable device of choice is Tomorrow Options WalkinSense[11] which contains eight pressure sensors on each insole and uses Bluetooth technology for transmission of data. The sensors were distributed on four main section of each insole with two sensors on each section to gather pressure data on the toes, metatarsal head, metatarsal arch and the heel. The configuration of the sensor is shown in Figure 2.

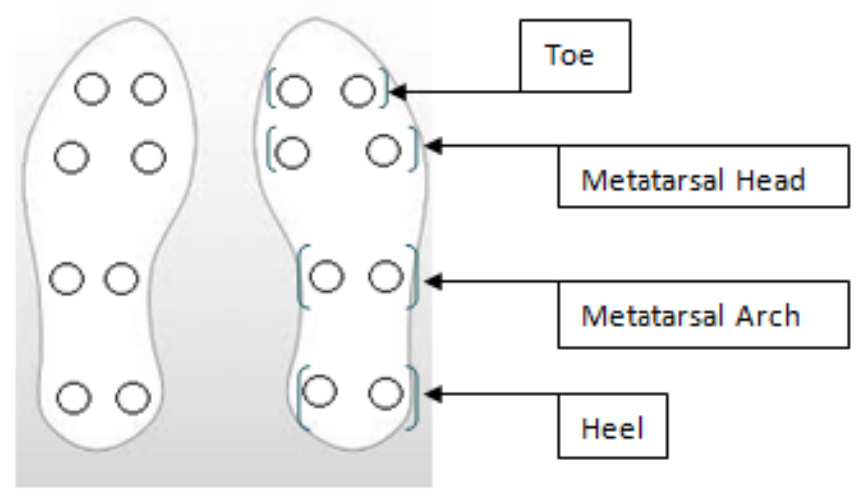

Fig 2. Smart insole sensor configuration used in this study to validate events in the acceleration data.

\section{B. Development and Interface Design}

In order to capture the raw accelerometer data of the iPhone, an application was developed. The iPhone development requires the Xcode development environment.
Xcode is an integrated development environment (IDE) for Mac OS X. The main functionalities of the application is to be able to gather the acceleration of each of the axes of the device while walking, adjustable sampling rate and formatting the data for transferring of data captured. Interface of the application uses UI elements of the interface builder provided by the IDE.

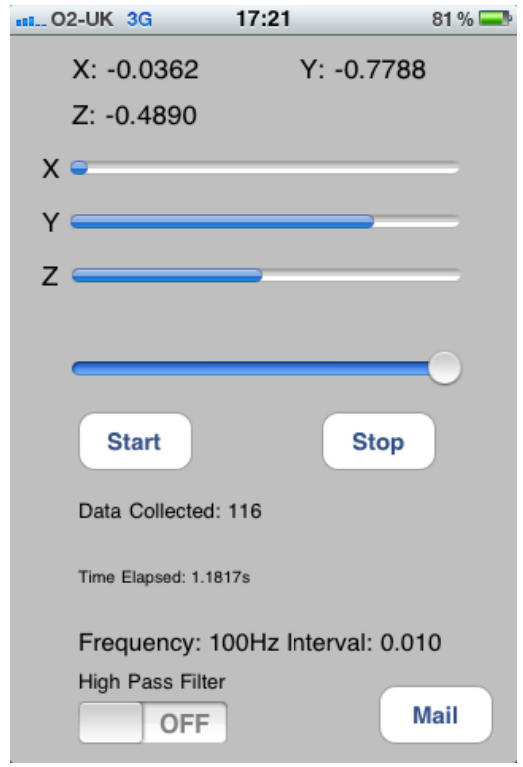

Fig 3. iPhone application interface.

\section{Data Collection}

The iPhone is placed on the left pocket to retrieve data whilst walking. In this placement position, the anteriorposterior acceleration is represented by the $\mathrm{X}$-axis, vertical acceleration by $\mathrm{Y}$-axis and medio-lateral acceleration by Zaxis.

Three different sample rates, $10 \mathrm{~Hz}, 60 \mathrm{~Hz}$, and $100 \mathrm{~Hz}$ were tested to determine whether the device was capable of operating at these frequencies and $100 \mathrm{~Hz}$ was the best choice. In this paper, the results are based on the $100 \mathrm{~Hz}$ sampling rate.

WalkinSense smart insoles were used whilst walking with the iPhone device in the left pocket. In order to synchronize different devices, a series of tasks such as walking, jumping and standing stationary for a period of time is used for synchronizing against the acceleration data and pressure data.

When collecting gait data, the subject walks at a distance of 10 meters on a flat surface with iPhone and wearing trainers with smart insoles.

\section{Analysis}

Matlab is used to visualise and analysis the data collected. The absolute distance algorithm [12] and step detection algorithm [13] was adapted to provide step detection of the acceleration data. A variation between steps was noted, the higher peak is when the left foot is in the swing phase and lower peak is during stance phase. This was distinguished as left step is the initial step of the walking experiment. The following is the description of the algorithm used for step detection. 


\section{Step Detection Algorithm}

1. Initialise: Extract $\mathrm{x}, \mathrm{y}, \mathrm{z}$-axis acceleration data

2. Filter Data: using Low-pass filter on each axis

3. Obtain magnitude of $x, y, z$-axis, get an array $\boldsymbol{m}$.

4. Apply Absolute Value Function to array $\boldsymbol{m}$.

5. Normalize array $\boldsymbol{m}$.

\section{Repeat}

Compare each element in the $\boldsymbol{m}$ array of data with its previous one and next data value, if this element is greater than the other two, a peak value found.

7. Store the peak value into an array peaks.

8. Until there is only one element in the array.

9. Calculate average peak value, Average $\boldsymbol{e}_{\text {peaks }}$ by calculating the average value of array peaks.

10. Input step number

11. Sort peaks array in descending order, peak value which its order number is equal to the input step number is the calculated threshold.

Absolute Distance Algorithm was used on the dataset gathered to determine peaks and underlying patterns along the dataset based on the variation of points on all three axis of the iPhone accelerometer. The magnitude is found by applying the formula:

$$
m_{i}=\sqrt{x_{i}{ }^{2}+y_{i}{ }^{2}+z_{i}{ }^{2}}, i=1, \ldots, k
$$

In the above formula, $m_{i}$ is the result magnitude of the three axes and $k$ is the number of data collected. Absolute value function is used to get the absolute value of each data point. Normalization is performed to standardize the data into a common scale.

Further analysis was carried out with the aid of iGait software. iGait is a Matlab based software developed at the University of Ulster[14] for extraction of gait features from acceleration data.

\section{E. Validation}

Insole pressure sensor was used alongside the data collection of the iPhone to validate the events within the acceleration data. During analysis of the anterior-posterior axis, the graph showed a recurring trough that represented the stride time of the gait pattern. To validate the theory of using the trough as indicator of stride time, the data from the Walkin Sense smart insoles were used to correlate patterns by synchronising the insole data with acceleration data.

\section{RESULTS}

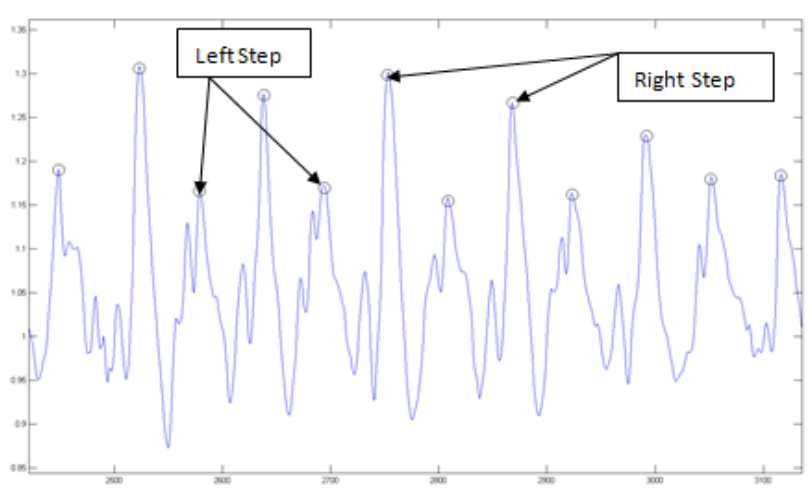

Fig 4. The magnitude of the tri-axial accelerometer data.

An evaluation of individual axis showed that noise was a major issue of the iPhone device. In order to reduce the noise, a low pass filter was applied onto the raw data generated from the device. The $\mathrm{X}$-axis after pre-processing displayed a recurring trough in the graph shown in Figure 5.

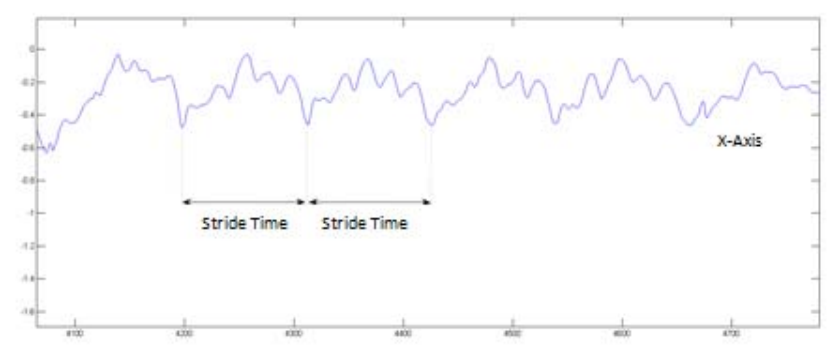

Fig 5. iPhone Accelerometer $\mathrm{X}$-axis representing anterior-posterior motion.

In Figure 6, the heel section of left insole is used to clearly identify heel contact of the left foot. The peaks represent the highest pressure exerted by the foot. The distance between each peak represent the stride time. The average time between the peaks from the smart insoles was found to be 1.15 seconds. The average time between the troughs of the $\mathrm{x}$-axis of the iPhone accelerometer was found to be 1.17 seconds. By using the trough of the acceleration data can provide a precise measurement of the stride time.

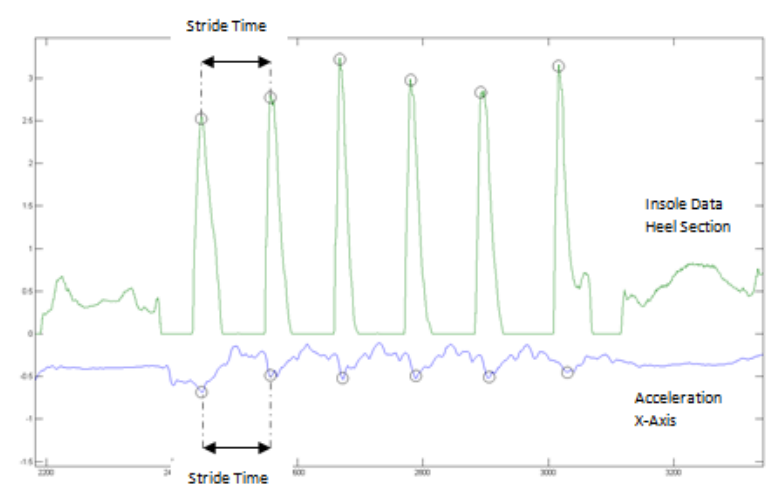


Fig 6. Insole Data synchronised with iPhone acceleration data by time.

An iGait study was conducted. A formatted data file from the iPhone acceleration data set was inserted into iGait for feature extraction. The software was capable of identifying steps and cadence from the acceleration data. Figure 6 shows the output of the data set from the iPhone device.

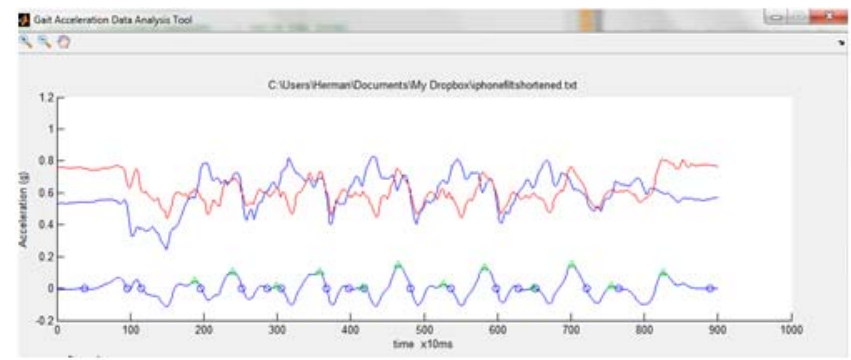

Fig 7. iGait results, which indicated when steps occurred.

\section{DISCUSSION}

The study provided an insight of the capabilities of the iPhone accelerometer. The result has validated the possibilities of using iPhone accelerometer to detect gait events such as stride time and step detection. The insole sensor device provided a clearer indication of gait events against the acceleration data. Further work with insole sensors should be able to provide information of heel contact and toe contact events within acceleration data.

There are limitations within the iPhone device such as positioning of the device. Positioning the device closer to the body center of mass could be a suitable position to consider. The device has limited memory and long period of recording acceleration data can cause the device to slow down and distort the dataset which was found in some initial development testing of the device.

Future work will look into the data collected to extract features of gait in different domains such as temporal, frequency domain, symmetry and regularity features. An experiment with positions of the device is the next step in the research to collaborate the data with iGait software for extraction of features. The overall aim of the research is to explore existing technologies in clinical environment, commercially available devices and wearable technologies. In reviewing the capabilities of smart technologies may be able to provide an alternative cost effective solution to monitoring gait of patients and the elderly to get along with their everyday activities.

\section{ACKNOWLEDGMENT}

This work is funded by Department for Employment \& Learning (DEL). The research is a part of the Smart Environment Research Group (SERG) in the University of Ulster.

\section{REFERENCES}

[1] Office for National Statistics (ONS), "Life Expectancy - Life expectancy continues to rise,' accessed Sept. 2010; http://www.statistics.gov.uk/cci/nugget.asp?id=168.

[2] D. L. Covington, J. G. Maxwell and T. V. Clancy, "Hospital resources used to treat the injured elderly at North Carolina trauma centers," J. Am. Geriatr. Soc., vol. 41, pp. 847-852, Aug, 1993.

[3] AgeUK, "Falls in the over 65 s cost NHS $£ 4.6$ million a day," accessed June. 2010; http://www.ageuk.org.uk/latest-press/archive/falls-over-65scost-nhs/

[4] A. Godfrey, K.M. Culhane and G.M. Lyons, "Comparison of the performance of the activPAL ${ }^{\mathrm{TM}}$ professional physical activity logger to a discrete accelerometer-based activity monitor," Med Eng Phys 29, pp. 930-934, 2006.

[5] Codamotion, "Movement Analysis With CODA", accessed Nov. 2010; http://www.codamotion.com/engineering-systems-overview.html

[6] Vicon, "Vicon Application Overview", accessed Dec 2010; http://www.vicon.com/applications/

[7] Qualisys, "Optical Motion Capture - Accurate tracking of any kind of motion," accessed Dec 2010; www.qualisys.com

[8] Minisun, "A Device for Analyzing Body Motion," IDEEA Overview, accessed Nov 2010; http://www.minisun.com/IDEEA_overview.asp

[9] M. Yang, H.Zheng, H. Wang, S. McClean, J. Hall, N. Harris, "Assessing accelerometer based gait features to support gait analysis for people with complex regional pain syndrome," Petra '10 Proceedings of $3^{\text {rd }}$ International Conference on Pervasive Teechnologies related to Assistive Environments, 2010.

[10] ST Products, "LIS302DL MEMS motion sensor", accessed Feb 2011; http://www.st.com/stonline/books/pdf/docs/12726.pdf

[11] [Tomorrow Options, "WalkinSense," accessed Nov 2010; http://www.tomorrow-options.com/en/walkinsense

[12] Y. Du, C. Chacon, and C. Zhang, "Gait Authentication on iPhone Devices," McGill University, ECSE-475 Design Project Report, June 15 th 2009

[13] Y. Huang, H. Zheng, C. Nugent, P. McCullagh, S. McDonough, "Activity Monitoring Using an Intelligent Mobile - A Validation Study," submitted to Petra 2011.

[14] M. Yang, H. Zheng, H. Wang, S. McClean and D. Newell. "iGAIT: An Interactive Accelerometer Based Gait Analysis System." accessed 2010; http://rosalind.infj.ulst.ac.uk/igait/iGait.html 